\title{
Molecular epidemiology, risk factor analysis and Comparison of diagnostic methods for Rapid Diagnosis of Fungal Pneumonia in Critically ill Cirrhotics
}

\author{
Pratibha Kale1, Vikas Khillan¹, L G Mitra², S K Sarin³. \\ 1) Department of Microbiology, 2) Department of Critical Care Medicine, \\ 3) Department of Hepatology, Institute of Liver and Biliary Sciences, New \\ Delhi.
}

Objectives:

Liver cirrhosis is associated with dysregulation of the immune system and increased susceptibility to fungal infections.

-Ventilator-associated pneumonia (VAP) is an important complication of mechanical ventilation (MV).

-Currently, the assessment of pneumonia, response to antibiotic therapy relies on the resolution of the same criteria used in the diagnosis.

-The use of biomarkers may potentially improve the early diagnosis of infections allowing earlier and better identification and treatment of patients with severe life threatening infections, but clinical evidence is lacking.

We aimed to study the major risk factors and molecular epidemiology of fungal pneumonia in critically ill cirrhotic patients. Also compared the rapid diagnostic methods and biomarkers for fungal pneumonia.

Methods :

-Single-center, prospective cohort study of 100 critically ill cirrhotic patients with fungal pneumonia between January-September 2017.

-Demographics, comorbidities, and laboratory variables were recorded.

- Comparative analysis of culture and biomarkers; Bronchoalveolar lavage and serum galactomannan, serum procalciton in were measured by ELISA and chemiluminescence assay on day 1 , day 3 and day 7 .

-The final outcome considered were mortality within one month after diagnosis or discharge of the patient with stable parameters.

Results:

Genotyping revealed;in two clusters, clinical isolates recovered from patients matched those recovered from air
Clinical Characateristics

\begin{tabular}{|l|c|c|c|c|}
\hline \multicolumn{1}{|c|}{ Characteristic } & Survivor & $\begin{array}{c}\text { Non } \\
\text { survivor }\end{array}$ & Total & $\begin{array}{c}\text { P } \\
\text { value }\end{array}$ \\
\hline No of subjects (\%) & \multicolumn{1}{|c|}{$(52)$} & $48(\%)$ & 100 & \\
\hline Age (years) & $49.8 \pm 19.5$ & $60.7 \pm 8.9$ & $55.2 \pm 16$ & 0.038 \\
\hline Gender M/F & $37 / 15$ & $38 / 10$ & $75 / 25$ & 0.12 \\
\hline APACHE II baseline & $20.3 \pm 6.9$ & $21.1 \pm 5.9$ & $20.7 \pm 4$. & 0.28 \\
\hline Prior Steroids & $10(27.7)$ & $26(72.2)$ & $36(36)$ & 0.02 \\
\hline $\begin{array}{l}\text { Prolonged } \\
\text { hospitalization }\end{array}$ & $22(34.3)$ & $42(65)$ & $64(64)$ & 0.05 \\
\hline Neutropenia & $14(26.2)$ & $24(9)$ & 38 & 0.03 \\
\hline $\begin{array}{l}\text { Diabetes } \\
\text { mellitus(\%) }\end{array}$ & $10(43.4)$ & $12(54.5)$ & 22 & 0.33 \\
\hline Aspergillus flavus & $10 / 50$ & $28 / 50$ & $38 \%$ & \\
\hline A. fumigatus & $2 / 50$ & $9 / 50$ & $11 \%$ & \\
\hline
\end{tabular}

COMPARISON OF BIOMARKERS

\begin{tabular}{|c|c|c|c|c|c|c|}
\hline & \multicolumn{2}{|c|}{ Day 1} & \multicolumn{2}{|c|}{ Day 3} & \multicolumn{2}{|c|}{ Day 7} \\
\hline & $\begin{array}{l}\text { Survivo } \\
\mathrm{r}\end{array}$ & $\begin{array}{l}\text { Non- } \\
\text { surviv } \\
\text { or }\end{array}$ & $\begin{array}{l}\text { Survivo } \\
\mathrm{r}\end{array}$ & $\begin{array}{l}\text { Non- } \\
\text { surviv } \\
\text { or }\end{array}$ & $\begin{array}{l}\text { Survivo } \\
\mathrm{r}\end{array}$ & $\begin{array}{l}\text { Non- } \\
\text { surviv } \\
\text { or }\end{array}$ \\
\hline $\begin{array}{l}\text { PCT } \\
\mathrm{ng} / \mathrm{ml}\end{array}$ & $\begin{array}{l}2.5 \\
+3.9\end{array}$ & $\begin{array}{l}4.6 \pm \\
8.3\end{array}$ & $\begin{array}{l}0.6 \pm \\
0.7\end{array}$ & $\begin{array}{l}5.7 \pm \\
5.1\end{array}$ & $\begin{array}{l}0.6 \pm \\
1.3\end{array}$ & $\begin{array}{l}8.4 \pm \\
1.3\end{array}$ \\
\hline p Value & \multicolumn{2}{|c|}{0.78} & \multicolumn{2}{|c|}{0.03} & \multicolumn{2}{|c|}{0.04} \\
\hline \multirow{2}{*}{$\begin{array}{r}\text { BAL } \\
\text { Galacto } \\
\text { mannan } \\
\text { index }\end{array}$} & $\begin{array}{l}1.22 \\
\pm 1.1\end{array}$ & $\begin{array}{l}3.39 \\
+\quad 1.2\end{array}$ & $\begin{array}{l}1.8 \pm \\
1.8\end{array}$ & $\begin{array}{l}2.34 \\
\pm 1.1\end{array}$ & $\begin{array}{l}1.17 \pm 1 \\
.8\end{array}$ & $\begin{array}{l}2.32 \pm 1 \\
.8\end{array}$ \\
\hline & \multicolumn{2}{|c|}{0.03} & \multicolumn{2}{|c|}{0.18} & \multicolumn{2}{|c|}{0.11} \\
\hline \multirow{2}{*}{$\begin{array}{r}\text { Serum } \\
\text { Galacto } \\
\text { mannan } \\
\text { index }\end{array}$} & $\begin{array}{l}0.3 \pm \\
0.8\end{array}$ & $\begin{array}{l}0.4 \pm \\
0.6\end{array}$ & $\begin{array}{l}0.5 \pm \\
0.1\end{array}$ & $\begin{array}{l}0.4 \pm \\
0.4\end{array}$ & $\begin{array}{l}0.3 \pm \\
0.6\end{array}$ & $\begin{array}{l}0.5 \pm \\
.08\end{array}$ \\
\hline & \multicolumn{2}{|c|}{0.12} & \multicolumn{2}{|c|}{0.34} & \multicolumn{2}{|c|}{0.23} \\
\hline
\end{tabular}

\section{Discussion}

Fungal pneumonia is a serious complication in cirrhotics with neutropenia, prolonged hospitalization and steroids as major risk factors. Aspergillus species predominate in consanguity with Asian epidemiology. Culture methods are reliable for diagnosis and combination with BAL galactomannan is useful for rapid diagnosis. Serum procalcitonin is raised in patients with fungal pneumonia. Higher procalcitonin values are associated with higher mortality and poor outcome in critically ill patients. In our study the baseline PCT at admission to ICU was higher in non- survivor group, levels on D3 and D7 were persistantly higher. High serum procalcitonin level and BAL Galactomannann are useful in fungal pneumonia to step up therapeutic measures. The observation of genetic relatedness of clinical and environmental sample might open new perspectives in the development of infection control measures to prevent invasive aspergillosis in high-risk patients. 